\title{
CADRE D'INDICATEURS DES MALADIES CHRONIQUES ET DES BLESSURES \\ STATISTIQUES RAPIDES, ÉDITION 2016
}

Diffuser cet article sur Twitter

\begin{tabular}{|c|c|c|c|}
\hline $\begin{array}{l}\text { GROUPE } \\
\text { D'INDICATEURS }\end{array}$ & MESURE(S) D'INDICATEUR & $\begin{array}{l}\text { DONNÉES LES } \\
\text { PLUS RÉCENTES }\end{array}$ & $\begin{array}{l}\text { SOURCE DE } \\
\text { DONNÉES (ANNÉE) }\end{array}$ \\
\hline \multicolumn{4}{|c|}{ DÉTERMINANTS SOCIAUX ET ENVIRONNEMENTAUX } \\
\hline Éducation & \% de la population âgée de 20 ans et plus n'ayant pas terminé ses études secondaires & $12,8 \%$ & $\operatorname{ESCC}(2014)$ \\
\hline Revenu & \% de la population vivant sous les seuils de faible revenu après impôt & $9,7 \%$ & ECR (2013) \\
\hline Emploi & $\begin{array}{l}\text { Taux de chômage annuel moyen (\% de la population active âgée de } 15 \text { ans et plus ne travaillant pas } \\
\text { pendant la période de référence) }\end{array}$ & $6,8 \%$ & EPA (2015) \\
\hline \multicolumn{4}{|c|}{ FACTEURS DE RISOUE ET DE PROTECTION EN BAS ÂGE } \\
\hline Allaitement maternel & $\begin{array}{l}\text { \% de femmes âgées de } 15 \text { et plus déclarant avoir nourri leur enfant exclusivement au sein pendant les six } \\
\text { premiers mois ou plus }\end{array}$ & $26,2 \%$ & ESCC (2011-2012) \\
\hline Poids à la naissance & \% de naissances vivantes associées à un faible poids à la naissance & $6,1 \%$ & SEC (2011) \\
\hline $\begin{array}{l}\text { Exposition à la fumée } \\
\text { secondaire }\end{array}$ & $\begin{array}{l}\text { \% de ménages avec enfants âgés de moins de } 12 \text { ans régulièrement exposés à la fumée de tabac } \\
\text { à la maison }\end{array}$ & $3,1 \%$ & ECTAD (2013) \\
\hline Violence familiale & $\begin{array}{l}\text { \% de la population ayant subi I'un des trois types de maltraitance (violence physique, agression sexuelle et } \\
\text { exposition à la violence conjugale) avant l'âge de } 16 \text { ans }\end{array}$ & $32,3 \%$ & ESCC-SM (2012) \\
\hline \multicolumn{4}{|c|}{ FACTEURS DE RISQUE ET DE PROTECTION COMPORTEMENTAUX } \\
\hline \multirow[t]{2}{*}{ Activité physique } & $\begin{array}{l}\text { \% d'enfants et de jeunes âgés de } 5 \text { à } 17 \text { ans qui pratiquent au moins } 60 \text { minutes d'activité physique } \\
\text { d'intensité modérée à élevée par jour }\end{array}$ & $9,3 \%$ & ECMS (2012-2013) \\
\hline & $\begin{array}{l}\text { \% de la population âgée de } 18 \text { ans et plus respectant les directives d'activité physique en pratiquant au } \\
\text { moins } 150 \text { minutes d'activité physique d'intensité modérée à élevée par semaine, par session de } 10 \text { minutes } \\
\text { ou plus }\end{array}$ & $22,2 \%$ & ECMS (2012-2013) \\
\hline \multirow[t]{3}{*}{$\begin{array}{l}\text { Comportement } \\
\text { sédentaire }\end{array}$} & $\begin{array}{l}\text { \% d'enfants et de jeunes âgés de } 5 \text { à } 17 \text { ans déclarant passer plus de } 2 \text { heures par jour à regarder la } \\
\text { télévision ou à utiliser l'ordinateur lors de leur temps libre }\end{array}$ & $48,2 \%$ & ECMS (2012-2013) \\
\hline & $\begin{array}{l}\text { Moyenne de temps quotidien consacré aux activités sédentaires (excluant les heures } \\
\text { de sommeil), population âgée de } 5 \text { à } 17 \text { ans }\end{array}$ & 8,5 heures & ECMS (2012-2013) \\
\hline & $\begin{array}{l}\text { Moyenne de temps quotidien consacré aux activités sédentaires (excluant les heures de sommeil) } \\
\text { population âgée de } 18 \text { ans et plus }\end{array}$ & 9,8 heures & ECMS (2012-2013) \\
\hline Saine alimentation & $\begin{array}{l}\text { \% de la population âgée de } 12 \text { ans et plus déclarant consommer des fruits et légumes au moins cinq fois } \\
\text { par jour }\end{array}$ & $39,7 \%$ & ESCC (2014) \\
\hline Alimentation malsaine & \% de la population âgée de 5 à 17 ans déclarant consommer quotidiennement des boissons sucrées & $17,2 \%$ & ECMS (2012-2013) \\
\hline Sommeil adéquat & $\begin{array}{l}\text { \% d'enfants et d'adolescents âgés de } 5 \text { à } 17 \text { ans déclarant bénéficier d'un nombre adéquat d'heures de } \\
\text { sommeil au quotidien (10 à } 13 \text { heures pour ceux de } 5 \text { ans, } 9 \text { à } 11 \text { heures pour ceux de } 6 \text { à } 13 \text { ans et } 8 \text { à } 10 \\
\text { heures pour ceux de } 14 \text { à } 17 \text { ans) }\end{array}$ & $74,6 \%$ & ECMS (2012-2013) \\
\hline \multirow{2}{*}{$\begin{array}{l}\text { Stress chronique et } \\
\text { capacité d'adaptation }\end{array}$} & \% de la population âgée de 18 ans et plus déclarant avoir une grande capacité d'adaptation & $56,9 \%$ & ESCC-SM (2012) \\
\hline & $\begin{array}{l}\text { \% de la population âgée de } 12 \text { ans et plus déclarant que la plupart de ses journées ont été } \\
\text { "assez stressantes » ou " extrêmement stressantes » au cours des } 12 \text { derniers mois }\end{array}$ & $22,4 \%$ & ESCC (2014) \\
\hline Consommation d'alcool & $\begin{array}{l}\text { \% de la population âgée de } 15 \text { ans et plus déclarant boire davantage d'alcool que ce que préconisent les } \\
\text { directives de consommation d'alcool à faible risque à long terme }\end{array}$ & $15,7 \%$ & ECTAD (2013) \\
\hline \multirow[t]{2}{*}{ Tabagisme } & \% de la population âgée de 15 ans et plus déclarant fumer (quotidiennement ou occasionnellement) & $14,6 \%$ & ECTAD (2013) \\
\hline & \% de la population âgée de 15 ans et plus déclarant fumer quotidiennement & $10,9 \%$ & ECTAD (2013) \\
\hline \multicolumn{4}{|l|}{ CONDITIONS À RISQUE } \\
\hline \multirow[t]{2}{*}{ Obésité } & \% d'enfants et de jeunes âgés de 5 à 17 ans qui sont obèses (donnée mesurée) & $12,5 \%$ & ECMS (2012-2013) \\
\hline & \% de la population âgée de 18 ans et plus qui est obèse (donnée mesurée) & $26,4 \%$ & ECMS (2012-2013) \\
\hline $\begin{array}{l}\text { Taux élevé de glucose } \\
\text { dans le sang }\end{array}$ & \% de la population âgée de 20 ans et plus ayant une glycémie élevéec (donnée mesurée) & $4,1 \%$ & ECMS (2012-2013) \\
\hline $\begin{array}{l}\text { Cholestérol sanguin } \\
\text { élevé }\end{array}$ & $\begin{array}{l}\text { \% de la population âgée de } 20 \text { ans et plus ayant un taux de cholestérol élevéc (ratio cholestérol total sur } \\
\text { cholestérol LHD [CT/C-LHD]) (donnée mesurée) }\end{array}$ & $16,8 \%$ & ECMS (2012-2013) \\
\hline Hypertension & Prévalence de l'hypertension au sein de la population âgée de 20 ans et plus & $24,9 \%$ & $\operatorname{SCSMC}(2011 / 12)^{d}$ \\
\hline \multicolumn{4}{|c|}{ PRATIQUES DE PRÉVENTION DES MALADIES } \\
\hline \multirow{2}{*}{$\begin{array}{l}\text { Contact avec un } \\
\text { professionnel de la } \\
\text { santé }\end{array}$} & $\begin{array}{l}\text { \% de la population âgée de } 12 \text { ans et plus déclarant avoir consulté un médecin de famille ou un } \\
\text { omnipraticien au moins une fois au cours des } 12 \text { derniers mois }\end{array}$ & $75,6 \%$ & $\operatorname{ESCC}(2014)$ \\
\hline & $\begin{array}{l}\text { \% de la population âgée de } 12 \text { ans et plus déclarant avoir consulté un dentiste, un hygiéniste dentaire ou un } \\
\text { orthodontiste au moins une fois au cours des } 12 \text { derniers mois }\end{array}$ & $66,9 \%$ & $\operatorname{ESCC}(2014)$ \\
\hline \multirow[t]{3}{*}{$\begin{array}{l}\text { Dépistage des } \\
\text { maladies }\end{array}$} & $\begin{array}{l}\text { \% de femmes âgées de } 50 \text { à } 74 \text { ans déclarant avoir subi au moins une mammographie au cours des cinq } \\
\text { dernières années }\end{array}$ & $83,5 \%$ & $\operatorname{ESCC}(2012)$ \\
\hline & $\begin{array}{l}\text { \% de femmes âgées de } 25 \text { à } 69 \text { ans déclarant avoir subi au moins un test PAP au cours des trois dernières } \\
\text { années }\end{array}$ & $79,7 \%$ & ESCC (2012) \\
\hline & $\begin{array}{l}\text { \% de la population âgée de } 50 \text { à } 74 \text { ans ayant subi au moins un test de recherche de sang occulte dans les } \\
\text { selles, une colonoscopie ou une sigmoïdoscopie au cours de la période recommandée }\end{array}$ & $51,1 \%$ & ESCC (2012) \\
\hline Vaccination (grippe) & $\begin{array}{l}\text { \% de la population âgée de } 12 \text { ans et plus atteinte d'un problème de santé chronique ayant déclaré avoir } \\
\text { reçu un vaccin antigrippal saisonnier au cours des } 12 \text { derniers mois }\end{array}$ & $50,4 \%$ & $\operatorname{ESCC}(2014)$ \\
\hline \multicolumn{4}{|c|}{ ÉTAT DE SANTÉ GLOBAL ET IMPACTS SUR LA SANTÉ } \\
\hline \multirow[t]{6}{*}{ Santé générale } & $\begin{array}{l}\text { \% de la population âgée de } 12 \text { ans et plus déclarant avoir une « très bonne " } \\
\text { ou une « excellente » santé }\end{array}$ & $59,1 \%$ & $\operatorname{ESCC}(2014)$ \\
\hline & $\begin{array}{l}\text { \% de la population âgée de } 12 \text { ans et plus déclarant avoir une « très bonne " } \\
\text { ou une « excellente » santé mentale }\end{array}$ & $71,2 \%$ & $\operatorname{ESCC}(2014)$ \\
\hline & Espérance de vie à la naissance & 83,0 ans & $\begin{array}{r}\operatorname{SCSMC}(2009 / 10 \text { à } \\
2011 / 12)\end{array}$ \\
\hline & Espérance de vie à 65 ans & 21,5 ans & $\begin{array}{r}\operatorname{SCSMC}(2009 / 10 \text { à } \\
2011 / 12)\end{array}$ \\
\hline & Espérance de vie ajustée en fonction de l'état de santé à la naissance & 72,6 ans & $\begin{array}{r}\operatorname{SCSMC}(2008 / 09 \text { à } \\
2010 / 11)\end{array}$ \\
\hline & Espérance de vie ajustée en fonction de l'état de santé à 65 ans & 16,4 ans & $\begin{array}{r}\operatorname{SCSMC}(2008 / 09 \text { à } \\
2010 / 11)\end{array}$ \\
\hline
\end{tabular}




\begin{tabular}{|c|c|c|c|}
\hline $\begin{array}{l}\text { GROUPE } \\
\text { D'INDICATEURS }\end{array}$ & MESURE(S) D'INDICATEUR & $\begin{array}{l}\text { DONNÉES LES } \\
\text { PLUS RÉCENTES }\end{array}$ & $\begin{array}{c}\text { SOURCE DE } \\
\text { DONNÉES (ANNÉE) }\end{array}$ \\
\hline \multirow[t]{19}{*}{ Morbidité - prévalence } & \% de la population âgée de 20 ans et plus atteinte d'au moins l'une des 10 principales maladies chroniques ${ }^{\circ}$ & $38,4 \%$ & ESCC (2014) \\
\hline & $\begin{array}{l}\text { \% de la population âgée de } 20 \text { ans et plus atteinte d'au moins une maladie chronique majeure (cancer, } \\
\text { diabète, maladies cardiovasculaires, maladies respiratoires chroniques) }\end{array}$ & $21,4 \%$ & ESCC (2014) \\
\hline & Prévalence du diabète chez les enfants et les jeunes âgés de 1 à 19 ans & $0,3 \%$ & $\operatorname{SCSMC}(2011 / 12)$ \\
\hline & Prévalence du diabète au sein de la population âgée de 20 ans et plus & $9,8 \%$ & $\operatorname{SCSMC}(2011 / 12)$ \\
\hline & Prévalence des maladies cardiovasculaires (auto-déclarées) au sein de la population âgée de 20 ans et plus & $6,2 \%$ & ESCC (2014) \\
\hline & Prévalence d'accidents vasculaires cérébraux au sein de la population âgée de 20 ans et plus & $2,7 \%$ & $\operatorname{SCSMC}(2011 / 12)$ \\
\hline & Prévalence de l'insuffisance cardiaque au sein de la population âgée de 40 ans et plus & $3,6 \%$ & $\operatorname{SCSMC}(2011 / 12)$ \\
\hline & Prévalence des cardiopathies ischémiques au sein de la population âgée de 20 ans et plus & $8,4 \%$ & $\operatorname{SCSMC}(2011 / 12)$ \\
\hline & Prévalence de l'asthme chez les enfants et les jeunes âgés de 1 à 19 ans & $15,3 \%$ & $\operatorname{SCSMC}(2011 / 12)$ \\
\hline & Prévalence de l'asthme au sein de la population âgée de 20 ans et plus & $9,5 \%$ & SCSMC (2011/12) \\
\hline & Prévalence des maladies pulmonaires obstructives chroniques au sein de la population âgée de 35 ans et plus & $9,6 \%$ & SCSMC (2011/12) \\
\hline & Prévalence de l'arthrite au sein de la population âgée de 20 ans et plus & $17,9 \%$ & ESCC (2014) \\
\hline & $\begin{array}{l}\text { Prévalence des maladies mentales ou des troubles liés à l'utilisation de substances au cours de la vie au } \\
\text { sein de la population âgée de } 15 \text { ans et plus }\end{array}$ & $33,3 \%$ & ESCC-SM (2012) \\
\hline & $\begin{array}{l}\text { Prévalence annuelle de l'utilisation des services de santé en raison de troubles mentaux chez les enfants et } \\
\text { les jeunes âgés de } 1 \text { à } 19 \text { ans }\end{array}$ & $8,7 \%$ & $\operatorname{SCSMC}(2011 / 12)$ \\
\hline & $\begin{array}{l}\text { Prévalence annuelle de l'utilisation des services de santé en raison de troubles mentaux chez les adultes } \\
\text { âgés de } 20 \text { ans et plus }\end{array}$ & $16,3 \%$ & $\operatorname{SCSMC}(2011 / 12)$ \\
\hline & Prévalence de troubles anxieux et de l'humeur chez les enfants et les jeunes âgés de 12 à 19 ans & $9,3 \%$ & $\operatorname{ESCC}(2014)$ \\
\hline & Prévalence de troubles anxieux et de l'humeur chez les adultes âgés de 20 ans et plus & $12,0 \%$ & ESCC (2014) \\
\hline & Prévalence de l'ostéoporose diagnostiquée au sein de la population âgée de 40 ans et plus & $11,6 \%$ & SCSMC (2011/12) \\
\hline & \% de la population ayant reçu un diagnostic de cancer au cours des 10 dernières années & $2,4 \%$ & RCC (1999-2008) \\
\hline \multirow[t]{14}{*}{ Morbidité - incidence } & Taux d'incidence du diabète chez les enfants et les jeunes âgés de 1 à 19 ans & 41,0 pour 100000 & SCSMC (2011/12) \\
\hline & Taux d'incidence du diabète au sein de la population âgée de 20 ans et plus & 790,5 pour 100000 & SCSMC (2011/12) \\
\hline & Taux d'incidence de l'asthme chez les enfants et les jeunes âgés de 1 à 19 ans & 1079,7 pour 100000 & SCSMC (2011/12) \\
\hline & Taux d'incidence de l'asthme au sein de la population âgée de 20 ans et plus & 353,2 pour 100000 & $\operatorname{SCSMC}(2011 / 12)$ \\
\hline & $\begin{array}{l}\text { Taux d'incidence des maladies pulmonaires obstructives chroniques au sein de la population âgée de } 35 \text { ans } \\
\text { et plus }\end{array}$ & 885,2 pour 100000 & SCSMC (2011/12) \\
\hline & Taux d'incidence de l'insuffisance cardiaque au sein de la population âgée de 40 ans et plus & 522,6 pour 100000 & $\operatorname{SCSMC}(2011 / 12)$ \\
\hline & Taux d'incidence des cardiopathies ischémiques au sein de la population âgée de 20 ans et plus & 631,0 pour 100000 & SCSMC (2011/12) \\
\hline & Taux d'incidence de l'infarctus aigu du myocarde au sein de la population âgée de 20 ans et plus & 220,0 pour 100000 & SCSMC (2011/12) \\
\hline & Taux de fractures de la hanche au sein de la population âgée de 40 ans et plus & 149,7 pour 100000 & SCSMC (2011/12) \\
\hline & Taux d'incidence de tous les cancers au sein de la population masculine & 438,0 pour $100000^{9}$ & RCC (2010) \\
\hline & Taux d'incidence de tous les cancers au sein de la population féminine & 368,0 pour $100000^{9}$ & RCC (2010) \\
\hline & Taux d'incidence des blessures non intentionnelles au sein de la population totale & 607,3 pour 100000 & BDMH (2010-2011) \\
\hline & Taux d'incidence des blessures intentionnelles auto-infligées au sein de la population totale & 47,4 pour 100000 & BDMH (2010-2011) \\
\hline & Taux d'incidence des blessures résultant d'une agression au sein de la population totale & 24,1 pour 100000 & BDMH (2010-2011) \\
\hline Multimorbidité & $\begin{array}{l}\text { \% de la population âgée de } 20 \text { ans et plus atteinte de plusieurs maladies chroniques } \\
\text { (au moins } 2 \text { de } 10 \text { maladies chroniques) }\end{array}$ & $14,8 \%$ & $\operatorname{ESCC}(2014)$ \\
\hline Incapacité & $\begin{array}{l}\text { \% de la population âgée de } 12 \text { ans et plus déclarant être limitée « parfois » ou « souvent » dans ses activités } \\
\text { en raison de problèmes de santé ou d'une maladie }\end{array}$ & $32,7 \%$ & $\operatorname{ESCC}(2014)$ \\
\hline \multirow[t]{8}{*}{ Mortalité } & $\begin{array}{l}\text { Taux de mortalité au sein de l'ensemble de la population attribuable à une maladie chronique majeure } \\
\text { (maladies cardiovasculaires, cancer, maladies respiratoires chroniques, diabète) }\end{array}$ & 469,9 pour 100000 & SEC (2012) \\
\hline & Taux de mortalité au sein de l'ensemble de la population attribuable aux maladies cardiovasculaires & 191,9 pour 100000 & SEC (2012) \\
\hline & Taux de mortalité au sein de l'ensemble de la population attribuable au cancer & 213,2 pour 100000 & SEC (2012) \\
\hline & Taux de mortalité au sein de l'ensemble de la population attribuable aux maladies respiratoires chroniques & 44,8 pour 100000 & SEC (2012) \\
\hline & Taux de mortalité au sein de l'ensemble de la population attribuable aux blessures non intentionnelles & 32,4 pour 100000 & SEC (2012) \\
\hline & Taux de mortalité au sein de l'ensemble de la population attribuable à un homicide & 1,4 pour 100000 & SEC (2012) \\
\hline & Taux de mortalité au sein de l'ensemble de la population attribuable au suicide & 11,3 pour 100000 & SEC (2012) \\
\hline & Taux de mortalité au sein de l'ensemble de la population attribuable au diabète & 20,0 pour 100000 & SEC (2012) \\
\hline \multirow[t]{5}{*}{ Mortalité prématurée } & $\begin{array}{l}\text { Probabilité de mourir (\%) entre } 30 \text { et } 69 \text { ans de maladies chroniques majeures (maladies cardiovasculaires, } \\
\text { cancer, maladies respiratoires chroniques, diabète) }\end{array}$ & $10,7 \%$ & SEC (2012) \\
\hline & Probabilité de mourir (\%) entre 30 et de 69 ans d'une maladie cardiovasculaire & $3,2 \%$ & SEC (2012) \\
\hline & Probabilité de mourir (\%) entre 30 et de 69 ans d'un cancer & $6,8 \%$ & SEC (2012) \\
\hline & Probabilité de mourir (\%) entre 30 et de 69 ans d'une maladie respiratoire chronique & $0,7 \%$ & SEC (2012) \\
\hline & Probabilité de mourir (\%) entre 30 et de 69 ans du diabète & $0,5 \%$ & SEC (2012) \\
\hline
\end{tabular}

Abréviations : BDMH, Base de données sur la morbidité hospitalière; cholestérol LHD, cholestérol à lipoprotéines de haute densité; CT, cholestérol total; ECMS, Enquête canadienne sur les mesures de la santé; ECR, Enquête canadienne sur le revenu; ECTAD, Enquête canadienne sur le tabac, l'alcool et les drogues; EPA, Enquête sur la population active; ESCC, Enquête sur la santé dans les collectivités canadiennes; RCC, Registre canadien du cancer; SCSMC, Système canadien de surveillance des maladies chroniques; SEC, Statistiques de l'état civil; SM, santé mentale.

Tous les taux pancanadiens présentés sont bruts sauf indication contraire.

' Les estimations concernant les comportements sédentaires diffèrent de celles présentées dans le CIMCB 2015 en raison d'un ajustement effectué pour tenir compte d'un changement de formatage d'une variable au cours de la collecte de données durant l'enquête.

c Cet indicateur mesure la population à niveau élevé de cette condition d'après une visite médicale, sans toutefois tenir compte du statut diagnostique (ex : les individus diagnostiqués ultérieurement ou bien dont la situation est stable en raison de traitement n'est pas mesurée).

¿ Les données de l'ECMS existent pour cet indicateur. Elles présentent les taux pancanadiens selon le diagnostic et le contrôle de l'hypertension.

e Les dix maladies chroniques sont les maladies du cœur, les accidents vasculaires cérébraux, le cancer, l'asthme, la maladie pulmonaire obstructive chronique, le diabète, I'arthrite, la maladie d'Alzheimer et autres démences, les troubles de l'humeur (dépression) et l'anxiété.

Les quatre principaux groupes de maladies chroniques sont le cancer, le diabète, les maladies cardiovasculaires (maladies cardiaques et accident vasculaire cérébral) et les maladies respiratoires chroniques (asthme et maladie pulmonaire obstructive chronique).

9 Les taux ont été normalisés en fonction de l'âge de la population au Canada en 1991.

Correspondance : Division de la surveillance et de l'épidémiologie, Centre de prévention des maladies chroniques, Agence de la santé publique du Canada,

785 avenue Carling, Ottawa (Ontario)

Citation suggérée : Centre de prévention des maladies chroniques, Agence de la santé publique du Canada. Cadre d'indicateurs des maladies chroniques et des

blessures : statistiques rapides, édition 2016. Ottawa (Ont.) : Agence de la santé publique du Canada; 2016.

Utilisez l'outil en ligne du Cadre d'indicateurs des maladies chroniques afin de voir la répartition de données additionnelles: http://infobase.phac-aspc.gc.ca/cdiif/?l=fra 